\title{
SURGE MOTION ON A FLOATING CYLINDER IN WATER OF FINITE DEPTH
}

\author{
DAMBARU D. BHATTA
}

Received 10 September 2002 and in revised form 16 October 2002

\begin{abstract}
We derived added mass and damping coefficients of a vertical floating circular cylinder due to surge motion in calm water of finite depth. This is done by deriving the velocity potential for the cylinder by considering two regions, namely, interior region and exterior region. The velocity potentials for these two regions are obtained by the method of separation of variables. The continuity of the solutions has been maintained at the imaginary interface of these regions by matching the functions and gradients of each solution. The complex matrix equation is numerically solved to determine the unknown coefficients. Some computational results are presented for different depth-to-radius and draft-to-radius ratios.
\end{abstract}

2000 Mathematics Subject Classification: 76B15, 35Q35, 35J05.

1. Introduction. Offshore technology has experienced a remarkable growth during the last forty years. At present, a wide variety of offshore structures is being used. Some of the uses of these structures are oil and gas recovery and ocean energy extraction. It is very important to develop methods of design and construction which will help to produce structures which are safe, functional, economical, and capable of resisting the forces induced by man and environment over a required period of time. In recent years, there has been a great interest in the hydrodynamic interactions between the structures due to the presence of wave motions. The rapid development of offshore structures has led to this interest. Prediction of the pressure distribution and resulting hydrodynamic forces on rigid structures immersed in water in the presence of a free surface is one of the most important research areas of many applied scientists and engineers. The forces exerted by surface waves on offshore structures such as offshore drilling rigs or submerged oil storage tanks are of important considerations in the design of large submerged or semisubmerged structures. The forces induced by surface waves as well as the forces induced by the dynamic response of a mooring vessel at sea are important factors in the determination of the resulting motion. The former quantities are generally referred to simply as wave forces or excitation forces while the latter are often characterized by radiation forces. Some other examples of such structures requiring motion analysis are articulated towers, guyed tower, and tension leg platform. 
A rigid floating structure may undergo six degrees of freedom: three translational and three rotational. Assuming a suitable coordinate system $O X Y Z$, the translational motions in the $x, y$, and $z$ directions are referred to as surge, sway, and heave, respectively; and the rotational motions about the $x, y$, and $z$ axes are referred to as roll, pitch, and yaw, respectively. Here the $z$ axis is considered to be vertically upwards from its still water level. Often the structure is restrained to have fewer degrees of freedom due to the type of mechanical connection used to fasten it to the seafloor.

Numerical results for the added mass and damping coefficients of semisubmerged two-dimensional heaving cylinders in water of finite depth were presented by Bai [1]. He showed that the added mass is bounded for all frequencies in water of finite depth. He studied the limits of the added mass and damping coefficients for high and low frequencies. Yeung [5] presented a set of theoretical added masses and damping coefficients for a floating circular cylinder in finite-depth water. Sabuncu and Calisal [3] obtained hydrodynamic coefficients for vertical cylinders at finite water depth. Williams and Abul-Azm [4] investigated the hydrodynamic interactions between the members of an array of floating circular cylinders which occur when one member undergoes prescribed forced oscillations. They presented numerical results for array configurations consisting of 2-6 cylinders. Numerical results for the added mass of bodies heaving at low frequency in water of finite depth were also presented by McIver and Linton [2].

We assume that the fluid is incompressible, the fluid motion is irrotational, and the waves are of small amplitude. Here we consider the coefficients related to the motion with one degree of freedom, namely, translational motion in the $x$ direction, that is, surge. In this paper, we present a systematic mathematical formulation and solution for the boundary value problem to obtain the added mass and damping coefficients for a circular cylinder with surge motion in water of finite depth. Computational results are obtained using Java programming language. These results are presented in graphical forms for various depth-to-radius and draft-to-radius ratios.

2. Mathematical formulation. We consider the radiation due to surge motion by a floating circular cylinder of radius $a$ in water of finite depth $h$. The geometry of the situation is depicted in Figure 2.1. The cylinder is assumed to be floating with a draft $b$ in water. Cylindrical coordinate system $(r, \theta, z)$ is assumed with $z$-axis vertically upwards, $r$ measured radially from the $z$-axis, and $\theta$ measured from the positive $x$-axis. Then, for an incompressible and inviscid fluid and for small-amplitude wave, we can introduce a velocity potential $\Phi(r, \theta, z, t)$ such that

$$
\nabla^{2} \Phi=0 .
$$




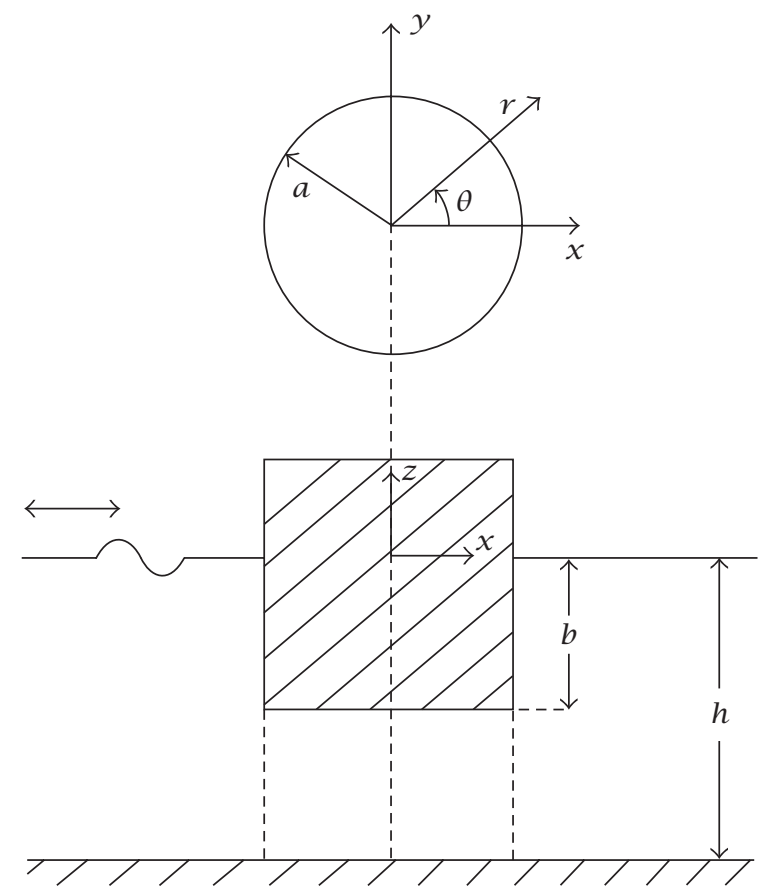

FIGURE 2.1. Definition sketch.

Free-surface boundary condition is

$$
\frac{\partial^{2} \Phi}{\partial t^{2}}+g \frac{\partial \Phi}{\partial z}=0 \quad \text { at } z=0
$$

Boundary condition at the sea bottom is

$$
\frac{\partial \Phi}{\partial z}=0 \quad \text { at } z=-h
$$

Body surface boundary condition at the bottom of the body is

$$
\frac{\partial \Phi}{\partial z}=0 \quad \text { on } r \leq a, z=-b
$$

Body surface boundary condition at the side of the body is

$$
\frac{\partial \Phi}{\partial r}=\frac{d \xi}{d t} \cos \theta \quad \text { at } r=a,-b \leq z \leq 0
$$

where $\xi$ is the surge displacement. 
The velocity potential $\Phi$ and the surge displacement $\xi$ can be written as

$$
\begin{aligned}
\Phi(r, \theta, z, t) & =\operatorname{Re}\left[\hat{\xi} \phi(r, \theta, z) e^{-i \sigma t}\right], \\
\xi & =\operatorname{Re}\left[\hat{\xi} e^{-i \sigma t}\right],
\end{aligned}
$$

where $\sigma$ is the angular frequency and $\hat{\xi}$ is the complex surge amplitude. Thus the boundary value problem reduces to

$$
\begin{gathered}
\nabla^{2} \phi=0, \\
g \frac{\partial \phi}{\partial z}-\sigma^{2} \phi=0 \quad \text { at } z=0, \\
\frac{\partial \phi}{\partial z}=0 \quad \text { at } z=-h, \\
\frac{\partial \phi}{\partial z}=0 \quad \text { on } r \leq a, z=-b, \\
\frac{\partial \phi}{\partial r}=-i \sigma \cos \theta \quad \text { at } r=a,-b \leq z \leq 0,
\end{gathered}
$$

and the radiation condition

$$
\lim _{r \rightarrow \infty} \sqrt{r}\left(\frac{\partial \phi}{\partial r}-i \lambda \phi\right)=0
$$

where $\lambda$ is an eigenvalue. We assume that $\phi$ takes the form

$$
\phi(r, \theta, z)=\sum_{m=0}^{\infty} \psi_{m}(r, z) \cos m \theta
$$

Now we obtain the interior solution and exterior solution. Superscripts $i$ and $e$ are used to denote the interior and exterior, respectively.

3. Interior solution. To obtain the interior solution for $\phi$, we write $\phi^{i}=$ $\sum_{m=0}^{\infty} \psi_{m}^{i}(r, z) \cos m \theta$. Then we have

$$
\begin{aligned}
& \nabla^{2} \psi_{m}^{i}-\frac{m^{2}}{r^{2}} \psi_{m}^{i}=0, \\
& \frac{\partial \psi_{m}^{i}}{\partial z}=0 \quad \text { at } z=-h \\
& \frac{\partial \psi_{m}^{i}}{\partial z}=0 \quad \text { on } z=-b
\end{aligned}
$$

Now, putting $Z^{\prime \prime} / Z=k_{0}^{2}$, we can write some possible solutions as ( $A \cosh k_{0} z+$ $\left.B \sinh k_{0} z\right) J_{m}\left(k_{0} r\right),(A z+B) r^{m}$, and $\left(A_{n} \cos k_{n} z+B_{n} \sin k_{n} z\right) I_{m}\left(k_{n} r\right)$. Here $J_{m}\left(k_{0} r\right)$ is the Bessel function of the first kind of order $m$ and $I_{m}\left(k_{n} r\right)$ is the modified Bessel function of the first kind of order $m$. 
Out of these solutions, the first one gives a trivial solution. From the second one, we get $Z=B r^{m}$, and the third one gives

$$
A_{n} \frac{\cos k_{n}(z+h)}{\cos k_{n} h} I_{m}\left(k_{n} r\right), \quad \text { where } k_{n}=\frac{n \pi}{h-b}, n=1,2, \ldots
$$

So, finally we have

$$
\psi_{m}^{i}=\frac{\alpha_{m 0}}{2}\left(\frac{r}{a}\right)^{m}+\sum_{n=1}^{\infty} \frac{\alpha_{m n} I_{m}\left(k_{n} r\right)}{I_{m}\left(k_{n} a\right)} \cos k_{n}(z+h),
$$

where $\alpha_{m n}$ 's are constants.

At $r=a$, we have

$$
\psi_{m}^{i}(a, z)=\frac{\alpha_{m 0}}{2}+\sum_{n=1}^{\infty} \alpha_{m n} \cos k_{n}(z+h) .
$$

Multiplying both sides of this equation by $(2 /(h-b)) \cos k_{n}(z+h)$ and then integrating both sides from $-h$ to $-b$ (and using the orthogonal property of the functions $\cos k_{n}(z+h)$ ), we get an expression for $\alpha_{m n}$ in the following form:

$$
\alpha_{m n}=\frac{2}{h-b} \int_{-h}^{-b} \psi_{m}^{i}(a, z) \cos k_{n}(z+h) d z
$$

4. Exterior solution. In this case, the boundary value problem is

$$
\begin{aligned}
& \nabla^{2} \psi_{m}^{e}(r, z)-\frac{m^{2}}{r^{2}} \psi_{m}^{e}(r, z)=0, \\
& g \frac{\partial \psi_{m}^{e}}{\partial z}-\sigma^{2} \psi_{m}^{e}=0 \quad \text { at } z=0, \\
& \frac{\partial \psi_{m}^{e}}{\partial z}=0 \quad \text { at } z=-h, \\
& \frac{\partial \psi_{m}^{e}}{\partial r}=-i \sigma \quad \text { at } r=a,-b \leq z \leq 0 .
\end{aligned}
$$

Now, putting $Z^{\prime \prime} / Z=\lambda_{0}^{2}$, we can write some possible solutions as $\left(A \cosh \lambda_{0} z+\right.$ $\left.B \sinh \lambda_{0} z\right) H_{m}^{(1)}\left(\lambda_{0} r\right),(A z+B) r^{-m}$, and $\left(A_{j} \cos \lambda_{j} z+B_{j} \sin \lambda_{j} z\right) K_{m}\left(\lambda_{j} r\right)$. Here $H_{m}^{(1)}\left(\lambda_{0} r\right)$ is the Hankel function of the first kind of order $m$ and $K_{m}\left(\lambda_{j} r\right)$ is the modified Bessel function of the second kind of order $m$. If $x$ is large in $H_{m}^{(1)}$ and $K_{m}(x), H_{m}^{(1)}$ and $K_{m}$ satisfy the radiation condition. Applying boundary 
conditions, we arrive at an expression

$$
\psi_{m}^{e}(r, z)=\frac{\beta_{m 0} H_{m}^{(1)}\left(\lambda_{0} r\right)}{H_{m}^{(1)}\left(\lambda_{0} a\right)} Z_{\lambda_{0}}(z)+\sum_{j=1}^{\infty} \frac{\beta_{m j} K_{m}\left(\lambda_{j} r\right)}{K_{m}\left(\lambda_{j} a\right)} Z_{\lambda_{j}}(z),
$$

where

$$
Z_{\lambda_{0}}(z)=N_{\lambda_{0}}^{-1 / 2} \cosh \lambda_{0}(z+h), \quad Z_{\lambda_{j}}(z)=N_{\lambda_{j}}^{-1 / 2} \cos \lambda_{j}(z+h),
$$

with

$$
N_{\lambda_{0}}=\frac{1}{2}\left(1+\frac{\sinh 2 \lambda_{0} h}{2 \lambda_{0} h}\right), \quad N_{\lambda_{j}}=\frac{1}{2}\left(1+\frac{\sin 2 \lambda_{j} h}{2 \lambda_{j} h}\right),
$$

$j=1,2, \ldots$ Now, at $r=a$, we have

$$
\psi_{m}^{e}(a, z)=\sum_{j=0}^{\infty} \beta_{m j} Z_{\lambda_{j}}(z)
$$

Multiplying both sides of this equation by $Z_{\lambda_{j}}(z) / h$ and then integrating both sides from $-h$ to 0 (and using the orthogonal property of the functions $Z_{\lambda_{j}}(z)$ ), we get an expression for $\beta_{m j}$ in the following form:

$$
\beta_{m j}=\frac{1}{h} \int_{-h}^{0} \psi_{m}^{e}(a, z) Z_{\lambda_{j}}(z) d z
$$

$j=0,1,2, \ldots$

5. Matching of the interior and exterior solutions at $r=a$. To preserve the continuity of the two solutions $\phi^{i}$ and $\phi^{e}$ at the imaginary interface $r=a$, it is required to satisfy

$$
\phi^{i}(a, \theta, z)=\phi^{e}(a, \theta, z),\left.\quad \frac{\partial \phi^{i}}{\partial r}\right|_{r=a}=\left.\frac{\partial \phi^{e}}{\partial r}\right|_{r=a}
$$

for $-h \leq z \leq-b$. Thus we have

$$
\begin{aligned}
\psi_{m}^{i}(a, z) & =\psi_{m}^{e}(a, z), \\
\left.\frac{\partial \psi_{m}^{i}}{\partial r}\right|_{r=a} & =\left.\frac{\partial \psi_{m}^{e}}{\partial r}\right|_{r=a},
\end{aligned}
$$

for $-h \leq z \leq-b$. Also, body surface condition, namely,

$$
\left.\frac{\partial \phi^{e}}{\partial r}\right|_{r=a}=-i \sigma \cos \theta
$$


that is,

$$
\left.\frac{\partial \psi_{m}^{e}}{\partial r}\right|_{r=a}=-i \sigma
$$

is to be satisfied. From (3.5) and condition (5.2),

$$
\begin{aligned}
\alpha_{m n} & =\frac{2}{h-b} \int_{-h}^{-b} \psi_{m}^{i}(a, z) \cos k_{n}(z+h) d z \\
& =\frac{2}{h-b} \int_{-h}^{-b} \psi_{m}^{e}(a, z) \cos k_{n}(z+h) d z \\
& =\frac{2}{h-b} \int_{-h}^{-b} \sum_{j=0}^{\infty} \beta_{m j} Z_{\lambda_{j}} \cos k_{n}(z+h) d z \\
& =2 \sum_{j=0}^{\infty} \beta_{m j} L_{n \lambda_{j}},
\end{aligned}
$$

where

$$
\begin{aligned}
L_{0 \lambda_{j}} & =\frac{1}{h-b} \int_{-h}^{-b} Z_{\lambda_{j}} d z, \\
L_{n \lambda_{j}} & =\frac{1}{h-b} \int_{-h}^{-b} Z_{\lambda_{j}} \cos k_{n}(z+h) d z .
\end{aligned}
$$

Thus

$$
\begin{aligned}
L_{n \lambda_{0}}= & \frac{1}{h-b} \int_{-h}^{-b} Z_{\lambda_{0}} \cos k_{n}(z+h) d z \\
& =\frac{N_{\lambda_{0}}^{-1 / 2}}{h-b} \int_{0}^{h-b} \cosh \lambda_{0} u \cos k_{n} u d u \\
& =\frac{(-1)^{n} N_{\lambda_{0}}^{-1 / 2}(h-b) \lambda_{0} \sinh \lambda_{0}(h-b)}{(h-b)^{2} \lambda_{0}^{2}+n^{2} \pi^{2}}, \\
L_{n \lambda_{j}}= & \frac{1}{h-b} \int_{-h}^{-b} Z_{\lambda_{j}} \cos k_{n}(z+h) d z \\
= & \frac{N_{\lambda_{j}}^{-1 / 2}}{h-b} \int_{0}^{h-b} \cos \lambda_{j} u \cos k_{n} u d u \\
= & \frac{(-1)^{n} N_{\lambda_{j}}^{-1 / 2}(h-b) \lambda_{j} \sin \lambda_{j}(h-b)}{(h-b)^{2} \lambda_{j}^{2}-n^{2} \pi^{2}},
\end{aligned}
$$

$n=0,1,2, \ldots, j=1,2, \ldots$ 
Now, from the gradient condition (5.3) and body surface condition (5.5), we have

$$
\begin{gathered}
\sum_{n=1}^{\infty} \alpha_{m n} G_{m n} \cos k_{n}(z+h)+\sum_{j=0}^{\infty} \beta_{m j} \mathscr{G}_{m j} Z_{\lambda_{j}}(z) \\
=\frac{m \alpha_{m 0}}{2} \text { for }-h \leq z \leq-b, \\
\sum_{j=0}^{\infty} \beta_{m j} \mathscr{G}_{m j} Z_{\lambda_{j}}(z)=-i \sigma a \text { for }-b \leq z \leq 0,
\end{gathered}
$$

where

$$
\begin{gathered}
G_{m n}=-\frac{k_{n} a I_{m}^{\prime}\left(k_{n} a\right)}{I_{m}\left(k_{n} a\right)}, \quad \varphi_{m 0}=\frac{\lambda_{0} a H_{m}^{(1)^{\prime}}\left(\lambda_{0} a\right)}{H_{m}^{(1)}\left(\lambda_{0} a\right)} \\
\mathscr{G}_{m n}=\frac{\lambda_{n} a K_{m}^{\prime}\left(\lambda_{n} a\right)}{K_{m}\left(\lambda_{n} a\right)} .
\end{gathered}
$$

Now, multiplying (5.9) by $Z_{\lambda_{l}}(z) / h, l=0,1,2, \ldots$, and integrating in the regions of validity and adding them, we get

$$
\begin{array}{r}
\sum_{n=1}^{\infty} \alpha_{m n} G_{m n} L_{n \lambda_{l}}+\sum_{j=0}^{\infty} \frac{h}{h-b} \beta_{m j} \varphi_{m j} \delta_{\lambda_{j} \lambda_{l}} \\
=\frac{m \alpha_{m 0}}{2} L_{0 \lambda_{l}}-\frac{h}{h-b} i \sigma a M_{\lambda_{l}},
\end{array}
$$

where

$$
M_{\lambda_{l}}=\int_{-b}^{0} \frac{Z_{\lambda_{l}}(z)}{h} d z
$$

Thus

$$
\begin{aligned}
& M_{\lambda_{0}}=\frac{N_{\lambda_{0}}^{-1 / 2}}{\lambda_{0} h}\left[\sinh \lambda_{0} h-\sinh \lambda_{0}(h-b)\right], \\
& M_{\lambda_{j}}=\frac{N_{\lambda_{j}}^{-1 / 2}}{\lambda_{j} h}\left[\sin \lambda_{j} h-\sin \lambda_{j}(h-b)\right],
\end{aligned}
$$

$j=1,2, \ldots$. Now, substituting the values of $\alpha_{m n}$ from (5.6) in (5.11), we get a system of equations

$$
\sum_{j=0}^{\infty} E_{l j} \gamma_{m j}=X_{m l},
$$


where

$$
\begin{gathered}
E_{l j}=-m L_{0 \lambda_{l}} L_{0 \lambda_{j}}+\frac{h}{h-b} G_{m j} \delta_{\lambda_{j} \lambda_{l}}+2 \sum_{n=1}^{\infty} G_{m n} L_{n \lambda_{l}} L_{n \lambda_{j}}, \\
X_{m l}=-\frac{h}{h-b} M_{\lambda_{l}}, \quad \gamma_{m j}=\frac{\beta_{m j}}{i \sigma a},
\end{gathered}
$$

$l=0,1,2, \ldots$

6. Added mass and damping coefficients. The radiated force $F$ can be written as the real part of $f e^{-i \sigma t}$, where $f$ is given by

$$
f=-i \rho \sigma a \int_{\theta=0}^{2 \pi} \int_{z=-b}^{0} \hat{\xi} \phi^{e}(a, \theta, z) \cos \theta d z d \theta
$$

This radiated force $F$ can be decomposed into components in phase with the acceleration and the velocity of the cylinder in the following way:

$$
F=-\left(\mu \frac{\partial^{2} \xi}{\partial t^{2}}+v \frac{\partial \xi}{\partial t}\right)
$$

where $\mu$ and $v$ are the added mass and damping coefficients due to surge, respectively. Hence the added mass $\mu$ and damping coefficients $v$ are given by

$$
\begin{aligned}
\mu+i \frac{\nu}{\sigma}= & -\frac{i \rho a}{\sigma} \int_{\theta=0}^{2 \pi} \int_{z=-b}^{0} \phi^{e}(a, \theta, z) \cos \theta d z d \theta \\
= & -\sum_{j=0}^{\infty} \frac{i \pi \rho a}{\sigma} \int_{-b}^{0} \beta_{1 j} Z_{\lambda_{j}}(z) d z \\
= & \pi \rho a^{2} h\left[\frac{N_{\lambda_{0}}^{-1 / 2} \gamma_{10}}{\lambda_{0} h}\left\{\sinh \lambda_{0} h-\sinh \lambda_{0}(h-b)\right\}\right. \\
& \left.\quad+\sum_{j=1}^{\infty} \frac{N_{\lambda_{j}}^{-1 / 2} \gamma_{1 j}}{\lambda_{j} h}\left\{\sin \lambda_{j} h-\sin \lambda_{j}(h-b)\right\}\right] .
\end{aligned}
$$

7. Numerical results. The complex matrix equation (5.14) is to be solved in order to determine the unknown coefficients. This infinite-order system is made finite to solve it numerically by rearranging as

$$
\sum_{j=0}^{N_{p}} \mathscr{E}_{l j} \gamma_{1 j}=\mathscr{X}_{1 l},
$$




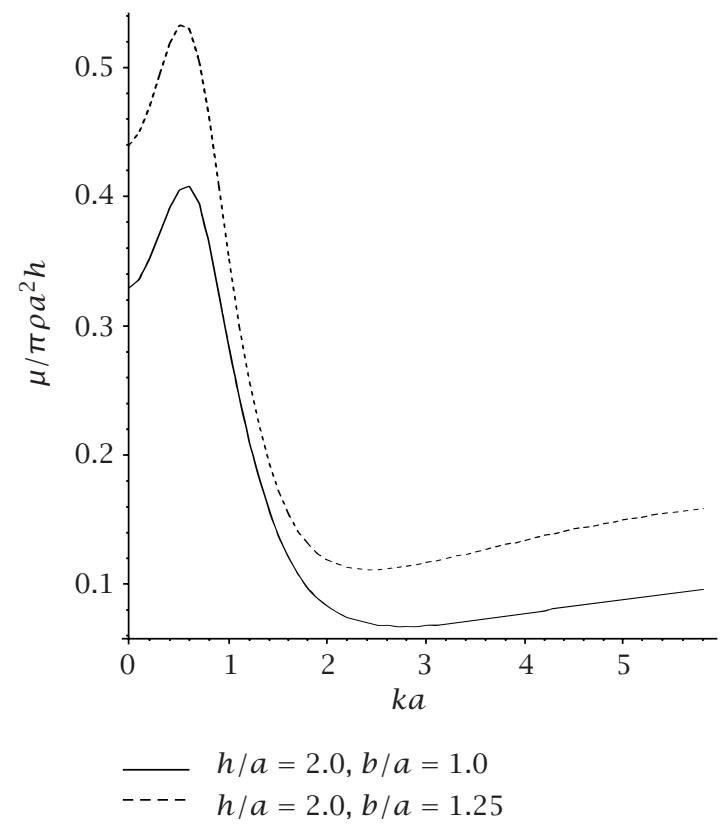

FIGURE 7.1. Nondimensional surge added mass for different $h / a$ and $b / a$.

where $\mathscr{E}_{l j}$ and $\mathscr{X}_{1 l}$ are given by

$$
\begin{gathered}
\mathscr{E}_{l j}=-L_{0 \lambda_{j}} L_{0 \lambda_{l}}+\frac{h}{h-b} \mathscr{G}_{1 j} \delta_{\lambda_{j} \lambda_{l}}+2 \sum_{n=1}^{N_{n}} G_{1 n} L_{n \lambda_{l}} L_{n \lambda_{j}}, \\
\mathscr{L}_{1 l}=-\frac{h}{h-b} M_{\lambda_{l}},
\end{gathered}
$$

$l=0,1, \ldots, N_{p}, j=0,1, \ldots, N_{p}$. Thus $\mathscr{E}$ is a square matrix of order $\left(N_{p}+1\right)$ and $\mathscr{X}$ is a vector of length $\left(N_{p}+1\right)$. Equation (7.1) is solved by using computer programs written in Java programming language. Once these $\gamma_{i j}$ 's are known, we can easily compute the nondimensional added mass and damping coefficients from the following:

$$
\begin{aligned}
\frac{\mu+i(\nu / \sigma)}{S}=[ & \frac{N_{\lambda_{0}}^{-1 / 2} \gamma_{10}}{\lambda_{0} h}\left\{\sinh \lambda_{0} h-\sinh \lambda_{0}(h-b)\right\} \\
& \left.+\sum_{j=1}^{\infty} \frac{N_{\lambda_{j}}^{-1 / 2} \gamma_{1 j}}{\lambda_{j} h}\left\{\sin \lambda_{j} h-\sin \lambda_{j}(h-b)\right\}\right],
\end{aligned}
$$

where $S=\pi \rho a^{2} h$.

Since the series appearing in (7.2) and (7.4) have an excellent truncation property, we have taken $N_{p}=10$ and $N_{n}=15$. Figures 7.1 and 7.2 show the 


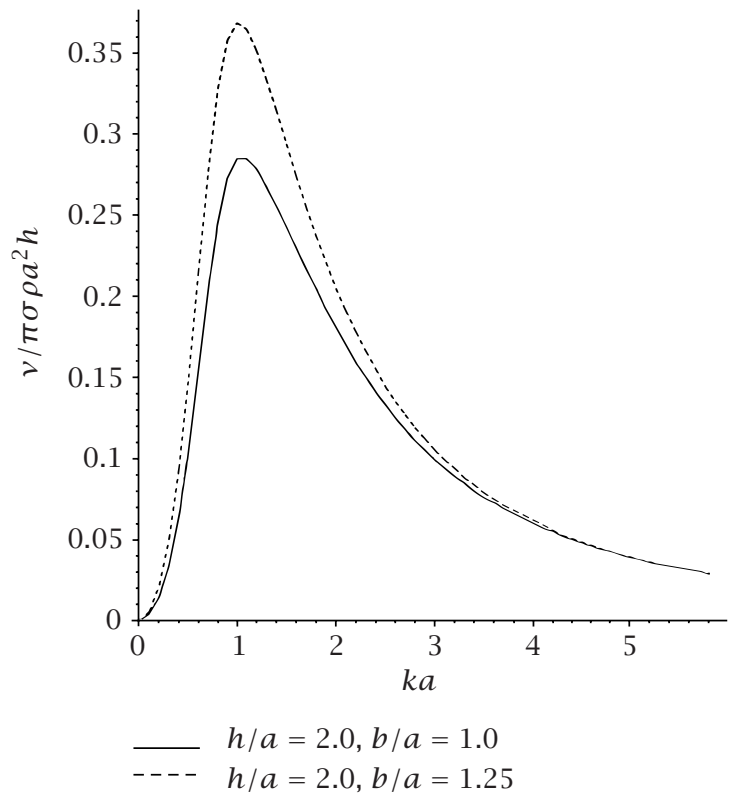

FIGURE 7.2. Nondimensional surge damping coefficients for different $h / a$ and $b / a$.

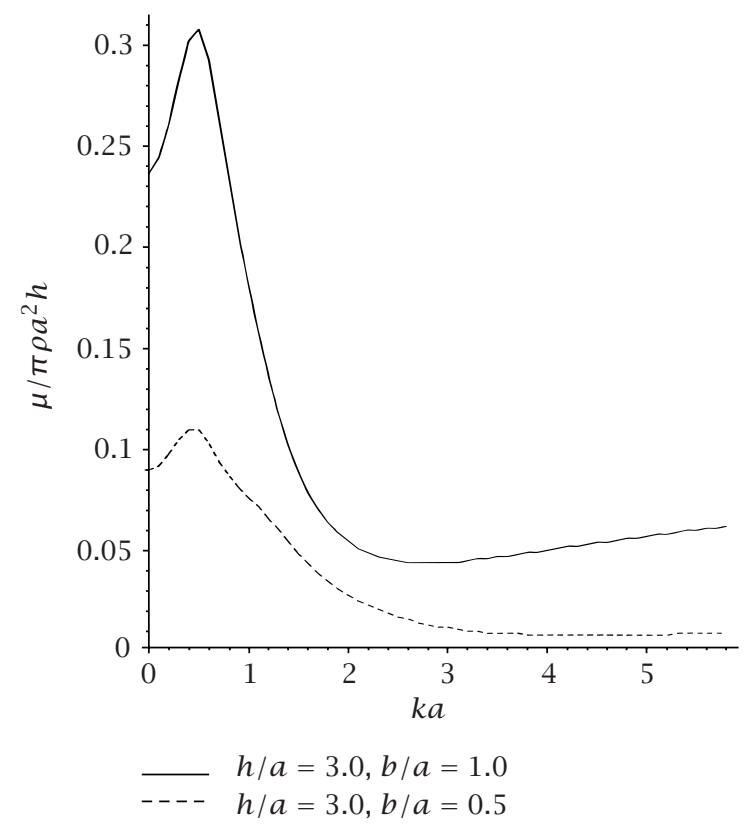

FIGURE 7.3. Nondimensional surge added mass for different $h / a$ and $b / a$. 


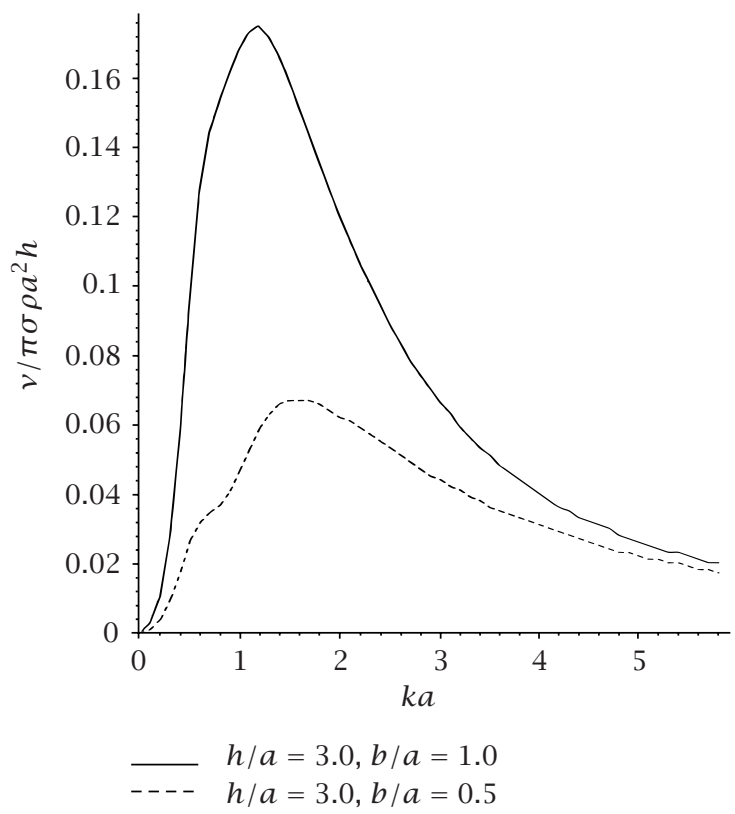

FIGURE 7.4. Nondimensional surge damping coefficients for different $h / a$ and $b / a$.

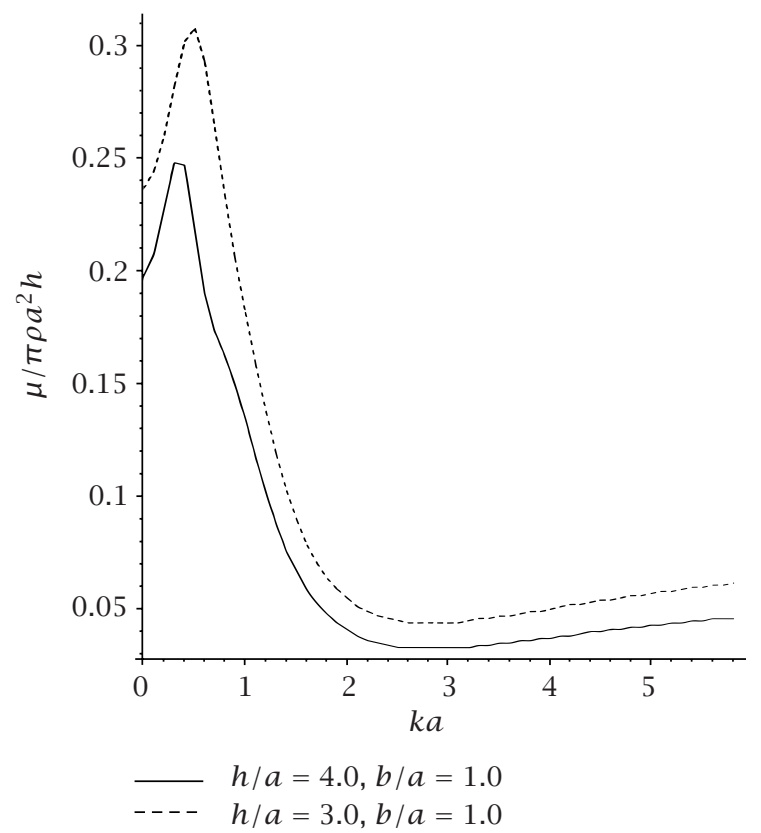

FIGURE 7.5. Nondimensional surge added mass for different $h / a$ and $b / a$. 


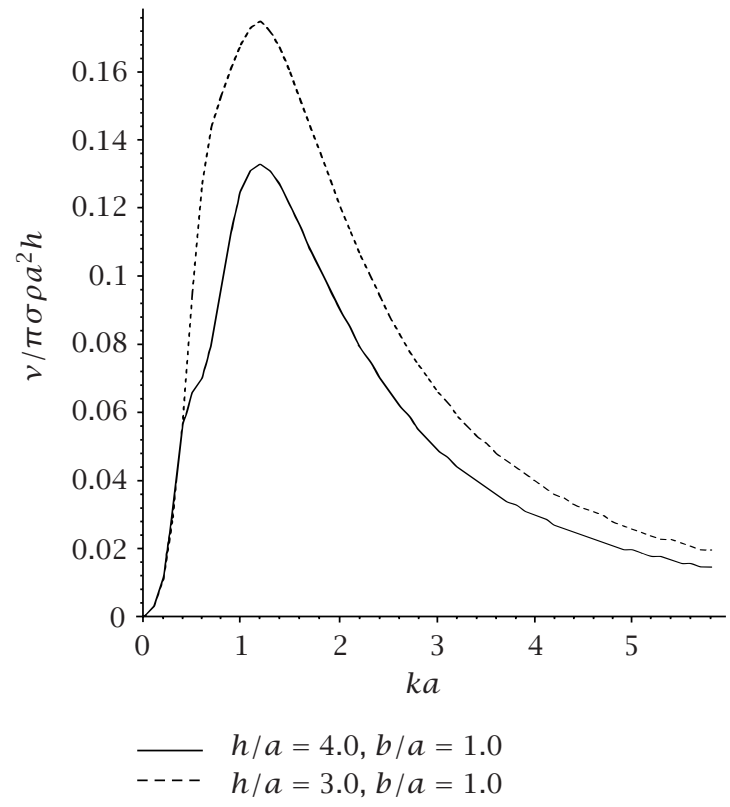

FiguRE 7.6. Nondimensional surge damping coefficients for different $h / a$ and $b / a$.

nondimensional added mass and damping coefficients for various $b / a$ when $h / a=2.00$, respectively. The nondimensional added mass and damping coefficients for various $b / a$ when $h / a=3.00$ are shown in Figures 7.3 and 7.4, respectively. Figures 7.5 and 7.6 show the nondimensional added mass and damping coefficients for various $h / a$ when $b / a=1.00$, respectively. From these numerical results, it is clear that nondimensional added mass and damping coefficients decrease with the decrease in draft at a fixed water depth for the same cylinder. Also for a fixed draft with the same cylinder, these coefficients decrease with the increase in water depth.

8. Conclusions. A mathematical treatment has been presented in this paper to derive the expressions for the velocity potential function by considering a floating circular cylindrical structure oscillating with surge motion in calm water of finite depth. Mathematical solutions for the boundary value problem are obtained in two physical regions, namely, interior region and exterior region, and matched at an imaginary interface to preserve the continuity of these two solutions. The added mass and damping coefficients are then computed using this potential. Results for different depth-to-radius and draft-to-radius ratios are presented in various figures. It can be concluded from the numerical results that nondimensional added mass and damping coefficients decrease with the decrease in draft at a fixed water depth for the same cylinder. Also, 
for a fixed draft with the same cylinder, these coefficients decrease with the increase in water depth.

\section{REFERENCES}

[1] K. J. Bai, The added mass of two-dimensional cylinders heaving in water of finite depth, J. Fluid Mech. 81 (1977), 85-105.

[2] P. McIver and C. M. Linton, The added mass of bodies heaving at low frequency in water of finite depth, Appl. Ocean Res. 13 (1991), no. 1, 12-17.

[3] T. Sabuncu and S. Calisal, Hydrodynamic coefficients for vertical circular cylinders at finite depth, Ocean Engng. 8 (1981), no. 1, 25-63.

[4] A. N. Williams and A. G. Abul-Azm, Hydrodynamic interactions in floating cylinder arrays-II. Wave radiation, Ocean Engng. 16 (1989), no. 3, 217-263.

[5] R. W. Yeung, Added mass and damping of a vertical cylinder in finite-depth waters, Appl. Ocean Res. 3 (1981), no. 3, 119-133.

Dambaru D. Bhatta: Department of Mathematics, Louisiana State University Alexandria (LSUA), Alexandria, LA 71302, USA

E-mail address: dbhatta@1sua.edu 


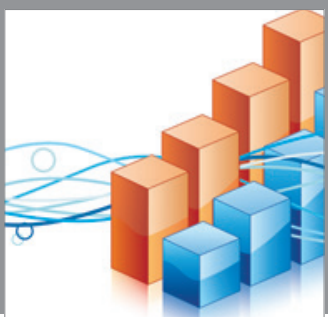

Advances in

Operations Research

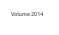

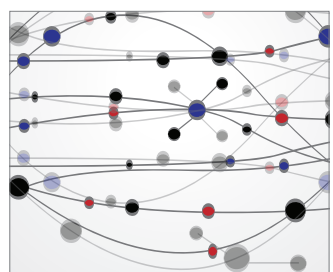

\section{The Scientific} World Journal
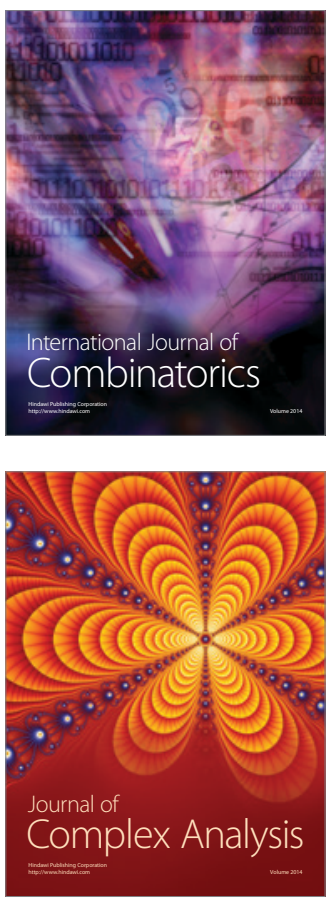

International Journal of

Mathematics and

Mathematical

Sciences
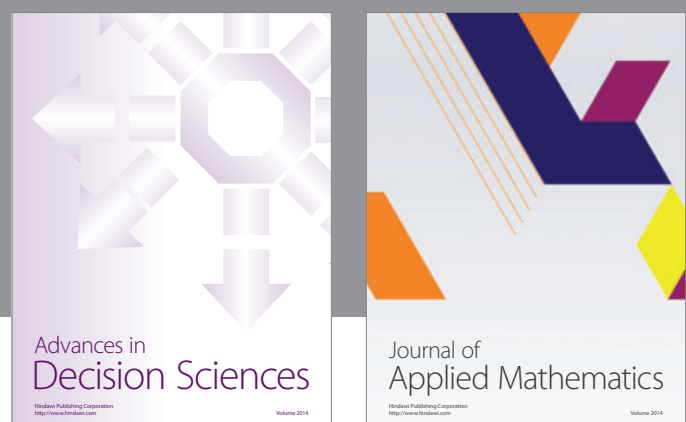

Journal of

Applied Mathematics
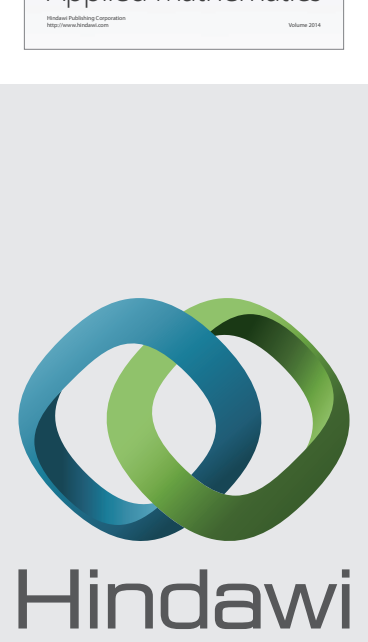

Submit your manuscripts at http://www.hindawi.com
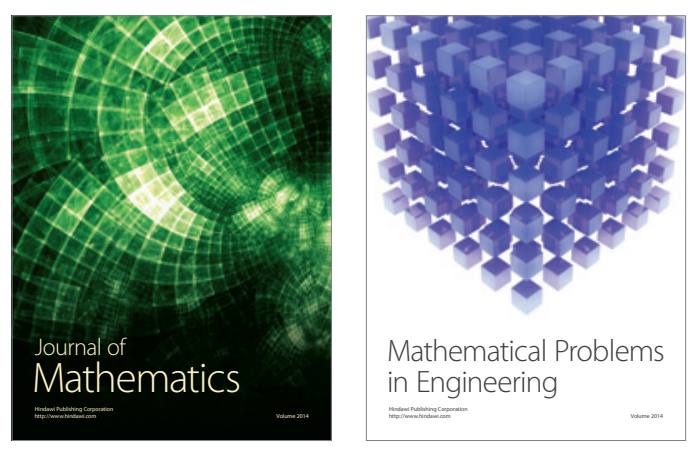

Mathematical Problems in Engineering
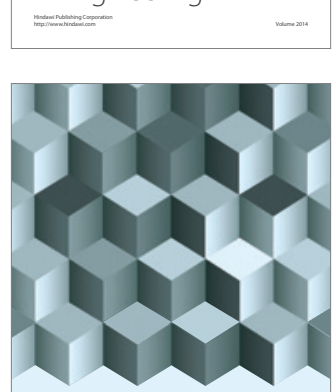

Journal of

Function Spaces
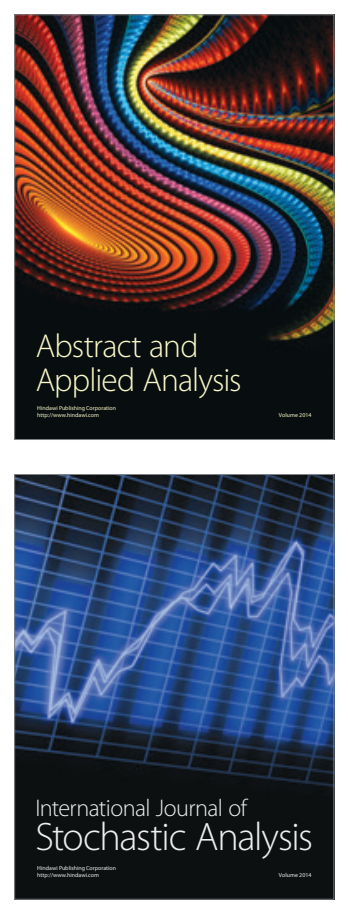

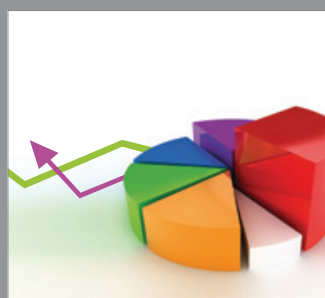

ournal of

Probability and Statistics

Promensencen
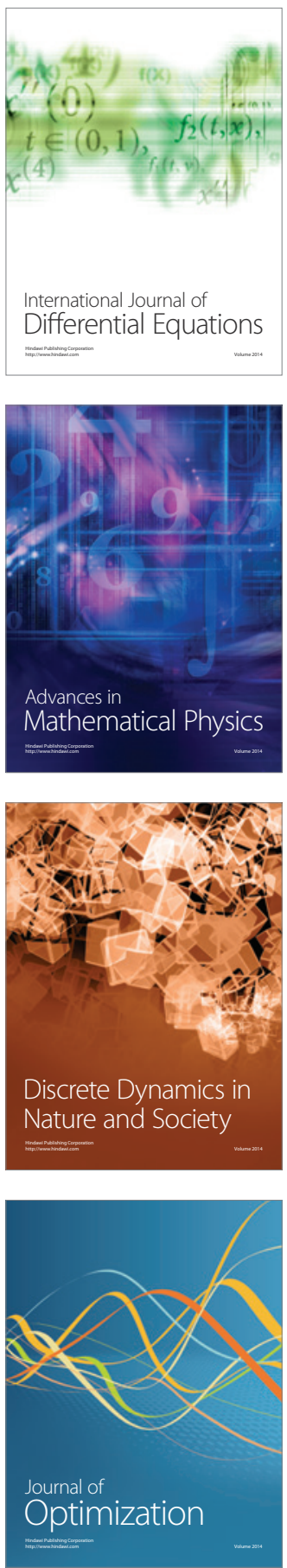